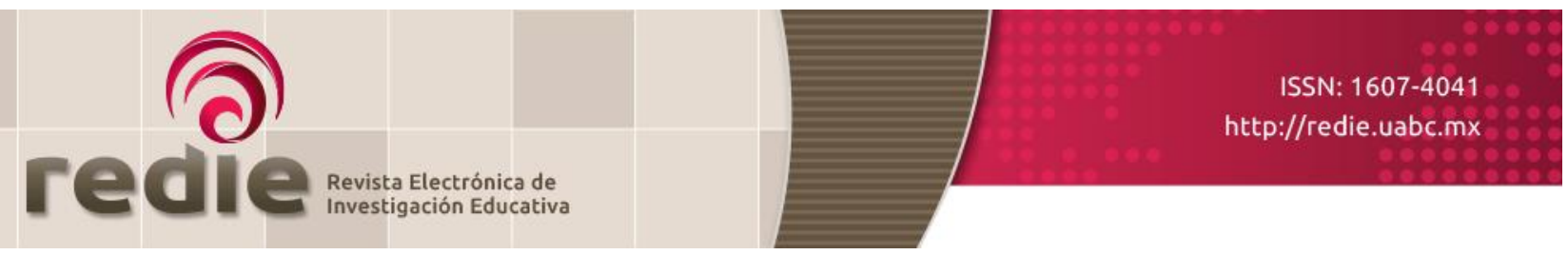

Vol. 19, Núm. 2, 2017

\title{
Variables académicas, comprensión lectora, estrategias y motivación en estudiantes universitarios
}

\section{Academic Variables, Reading Comprehension, Strategies and Motivation in University Students}

\author{
Jorge Guerra García (*) guerra@unam.mx \\ Carmen Yolanda Guevara Benitez (*) yolaguevara@hotmail.com \\ (*) Universidad Nacional Autónoma de México \\ (Recibido: 23 de marzo de 2015; Aceptado para su publicación: 13 de noviembre de 2015)
}

Cómo citar: Guerra, J. y Guevara, C. Y. (2017). Variables académicas, comprensión lectora, estrategias y motivación en estudiantes universitarios. Revista Electrónica de Investigación Educativa, 19(2), 78-90. https://doi.org/10.24320/redie.2017.19.2.1125

\section{Resumen}

El objetivo fue evaluar diversos niveles de comprensión lectora en estudiantes universitarios, relacionándolos con el uso de estrategias y motivación, y variables sociodemográficas y académicas. La muestra de 570 participantes se obtuvo por muestreo probabilístico estratificado de tipo proporcional. Se aplicaron dos instrumentos validados y un cuestionario. Los datos se analizaron con pruebas estadísticas ANOVA y $t$ de Student. Los resultados indicaron promedios alrededor del $66 \%$ de ejecución en comprensión y del 69\% en estrategias lectoras y motivación; el mayor porcentaje de alumnos dedicó entre seis y diez horas semanales a la lectura. Se obtuvieron diferencias estadísticamente significativas relacionadas con el grado académico: no contar con un empleo, promedio académico, contar con beca, género de los participantes, haber presentado exámenes extraordinarios y, en especial, con el tiempo dedicado a la lectura. El coeficiente Pearson arrojó una correlación positiva entre comprensión lectora y estrategias de lectura y motivación.

Palabras clave: Comprensión lectora, estrategias de lectura, motivación, variables académicas, estudiantes universitarios.

\section{Abstract}

The objective was to assess different levels of reading comprehension in university students and associate them with the use of strategies and motivation, and sociodemographic and academic variables. The sample of 570 participants was obtained through proportional stratified probability sampling. Two validated instruments and a questionnaire were applied. Data was analyzed using the ANOVA and Student's $t$ tests. Results showed mean values around $66 \%$ in comprehension and $69 \%$ in reading strategies and motivation; students that spent between six and ten hours a week reading made up the highest percentage. Statistically significant differences were observed with respect to the student's year: not having a job, average grade, having a scholarship, participants' sex, having taken make-up exams and, in particular, time spent reading. Pearson's coefficient produced a positive correlation between reading comprehension and reading strategies and motivation. 
Keywords: Reading comprehension, reading strategies, motivation, academic variables, collage students.

\section{Introducción}

La comprensión lectora ha adquirido relevancia en todo el mundo, hasta ser considerada una de las competencias genéricas más importantes a nivel formativo (Washer, 2007) e incluida dentro de las evaluaciones de desempeño internacionales y nacionales, como lo son el Instituto Nacional para la Evaluación de la Educación (INEE, 2009) y la Organización para la Cooperación y el Desarrollo Económico (OCDE, 2002).

La lectura se entiende como una interacción entre el texto y el lector, en la cual el lector asigna significado personal a lo que el autor pretende comunicar. El proceso de inferencia que establece el lector a partir de lo que lee, el uso que hace de diversas estrategias de lectura, así como la valoración que hace de los contenidos, son fundamentales para construir una versión individual del texto o, en otras palabras, para comprenderlo. De acuerdo con Pérez (2005), la comprensión se desarrolla en varios niveles: el primero, de comprensión literal, definido en función de reconocer y recordar el contenido; el segundo es el de reorganización de la información, cuando el lector ordena las ideas mediante clasificación y síntesis; el tercero es el inferencial, en donde el lector hace uso de su experiencia y conocimientos previos en relación con el tema, para realizar conjeturas o suposiciones; el cuarto corresponde a la lectura crítica o juicio valorativo que hace el lector, reflexionando sobre el contenido del texto; el último, de apreciación lectora, se refiere al impacto psicológico y estético que tiene el texto en el lector.

La comprensión se encuentra estrechamente relacionada con el concepto de estrategias lectoras, que se definen como el conjunto de acciones que regulan la conducta del lector, permitiéndole seleccionar, evaluar, persistir o abandonar dichas acciones según se alcance o no la comprensión (Peña, 2000).

Existen estrategias lectoras que han resultados ser efectivas para la comprensión, entre ellas: el uso del conocimientos previos sobre los temas del texto, para lograr la representación coherente y organizada del material leído (Taboada, Tonks, Wigfield y Guthrie, 2009); la identificación y representación de las ideas principales de un texto, a través de la elaboración de resúmenes (Wang, 2009), de organizadores gráficos (Oliver, 2009) o resaltando apropiadamente las ideas en el texto (Silvers, Kreiner y Natz-González, 2009); la identificación adecuada del tipo de texto (narrativo, expositivo u otro) para predecir su estructura general (Wang, 2009), y la habilidad del lector para hacerse preguntas relacionadas con el texto que lee -auto cuestionamiento- (Taboada et al., 2009). Lo ideal para lograr la comprensión es utilizar todas las tácticas mencionadas, que en conjunto se denominan estrategias metacognoscitivas, y que incluyen seleccionar la manera idónea de abordar cada lectura y completar el proceso con el automonitoreo y la autoevaluación del grado de efectividad de las estrategias utilizadas, lo que le permitirá al lector continuar con ellas o remplazarlas (Zhang y Wu, 2009).

Otro aspecto asociado estrechamente con la comprensión lectora y con el uso de estrategias es el relativo a la motivación del individuo hacia la lectura. Una persona que lee por gusto tiene una motivación intrínseca, mientras que la motivación extrínseca la muestran aquellos que leen para cumplir requisitos como la acreditación de materias. Anmarkrud y Braten (2009) demostraron que los estudiantes que se fijan metas de aprendizaje (de dominio o centradas en la tarea) están motivados intrínsecamente y, como un círculo virtuoso, utilizan estrategias que les permiten comprender los textos y lograr el aprendizaje o la comprensión. También hay relaciones positivas entre la percepción que tiene el individuo de sus propias habilidades para comprender textos y su ejecución en comprensión lectora; cuando se percibe capaz lleva a cabo estrategias efectivas para comprender.

Mujica, Guido y Mercado (2011) reportan que los alumnos universitarios suelen tener mayor motivación hacia la lectura de textos académicos cuando la tienen también por la lectura de otros temas, porque el disfrute que suscita la lectura es uno de los aspectos que motivan el comportamiento lector. Estos autores también señalan que las bajas puntuaciones de los alumnos mexicanos en pruebas internacionales guardan una estrecha relación con los bajos índices de actividades lectoras en la 
población, tomando como base los datos reportados en la Encuesta Nacional de Lectura. Entre los datos relevantes de dicha encuesta (período 2006-2012), destacan: un promedio anual de lectura de 2.94 libros por persona, que en el $56 \%$ de los hogares se tenían entre uno y diez libros, que la mayoría de la población prefería dedicarse a otras actividades recreativas por el escaso interés en la lectura, y que 33\% de los mexicanos nunca había leído un libro.

Estudios realizados en diversos países de Latinoamérica (Carlino, 2007; Cisneros, Olave y Rojas, 2012; Echevarría, 2006) han identificado varios problemas de comprensión lectora en estudiantes universitarios, específicamente con textos expositivo-argumentativos, que son los más utilizados en las materias escolares. Sus resultados indican que los problemas se presentaron, en mayor medida, en la selección y jerarquización de las ideas principales, el uso de la inferencia y la interpretación de lo leído. Tales hallazgos coinciden con los reportados para estudiantes mexicanos (Rizo, 2004; Sánchez y Acle, 2001).

Como se aprecia por lo antes expuesto, son varios los aspectos que pueden estar relacionados con la comprensión lectora de los estudiantes universitarios (nivel de comprensión que logran, tipo de texto que leen, estrategias que utilizan, motivación hacia la lectura y prácticas lectoras). Diversas investigaciones han aportado datos acerca de algunos de esos aspectos, pero no se han evaluado en una misma población de estudiantes, por lo que ha quedado sin estudiar la relación que guardan entre sí y la manera en que se asocian con variables sociodemográficas y académicas, tales como promedio escolar, tiempo dedicado al estudio y a la lectura, semestre de la carrera que se cursa, entre otras. Es necesario estudiar la relación que guardan todas esas variables entre sí en una misma población estudiantil.

El objetivo de la presente investigación fue evaluar, en una muestra de estudiantes universitarios, los niveles de comprensión lectora de un texto académico (expositivo-argumentativo), y relacionarlos con el uso de estrategias lectoras, la motivación hacia la lectura, así como con diversas variables sociodemográficas y académicas. Asimismo, observar si se presentan diferencias en dichos aspectos cuando los alumnos cursaban diferentes grados (semestres) de la carrera universitaria.

\section{Método}

El estudio fue de tipo no experimental, transversal, descriptivo y comparativo (Méndez, Namihira, Moreno y Sosa, 2006). Participaron 570 alumnos de la carrera de Psicología, distribuidos en 19 grupos de dos turnos (matutino y vespertino), inscritos en cuatro semestres escolares (10., 30., 50. y 70.) de una universidad pública mexicana. Para seleccionarlos se utilizó un muestreo probabilístico estratificado de tipo proporcional (Méndez et al., 2006), considerando semestre y turno de la población universo.

Se utilizó el Instrumento para medir Comprensión Lectora en Alumnos Universitarios (ICLAU), que consta de un texto expositivo-argumentativo de 965 palabras que explica la evolución biológica, haciendo énfasis en sus causas y procesos, e incluye siete reactivos para evaluar los cinco niveles de comprensión; las respuestas a los reactivos se evalúan a través de rúbricas, a excepción de la comprensión literal, que se evalúa por opción múltiple.

El proceso de conformación y obtención de la validez de contenido del ICLAU fue reportado por Guerra y Guevara (2013), quienes aclaran que para conformar la prueba se optó por este escrito debido a que toca un tópico general de biología, no vinculado directamente con la psicología; con ello, se procuró que fuera similar el nivel de conocimientos previos que pudieran tener los alumnos sobre dicho tema, independientemente del semestre que cursaran. Los aspectos validados en cada reactivo del instrumento fueron: redacción, pertinencia y adecuación en todos los elementos incorporados, incluyendo preguntas, opciones de respuesta, rúbricas y criterios de logro de cada nivel de comprensión lectora a evaluar (el Anexo I muestra ejemplos de reactivos).

También se aplicó el Inventario de Estrategias Metacognoscitivas y Motivación por la lectura (IEMML), diseñado y validado por Guerra, Guevara y Robles (2014) para aplicarse a estudiantes universitarios mexicanos, consta de 27 reactivos que se responden con base en una escala tipo Likert de cinco categorías (nunca, rara vez, a veces, frecuentemente y siempre). El inventario evalúa: 1) Estrategias de 
análisis de la lectura, incluye relacionar los contenidos de un texto con otros revisados antes o con experiencias vividas, parafrasear lo leído, reflexionar sobre el tema, elaborar nuevas ideas y conclusiones. 2) Estrategias para identificación de información o de ideas, se refiere a asegurarse de conocer el significado de los términos utilizados en el texto, ubicar las ideas principales y comprender su contenido. 3) Estrategias de consulta de fuentes de información, con reactivos que evalúan la búsqueda del significado de palabras y la consulta de nuevas fuentes bibliográficas. 4) Motivación intrínseca por la lectura en general, relacionada con actividades de lectura de cualquier tipo de texto, por iniciativa propia, y 5) Motivación intrínseca por la lectura de textos académicos, que se relacionan con el interés de los alumnos hacia este tipo de textos (el Anexo II muestra ejemplos de estos reactivos).

Por último, se utilizó un formato de auto reporte de datos sociodemográficos y académicos, para identificar en los alumnos: sexo, edad, estado civil, contar o no con un empleo remunerado, número de horas que labora al mes, turno en el que cursa sus estudios (matutino o vespertino), tiempo invertido semanalmente en la lectura de textos académicos, promedio académico, haber presentado exámenes extraordinarios, y contar o no con una beca académica.

Previa plática y planeación con los profesores de los grupos participantes, un equipo de psicólogos aplicó los instrumentos a cada grupo de alumnos, en el aula a la que asisten a sus clases. Al inicio de la sesión se les explicó en qué consistía la investigación, que los resultados no repercutirían en sus calificaciones académicas, y que podían incorporarse voluntariamente. A los alumnos que decidieron participar se les proporcionaron los formatos de consentimiento informado y los instrumentos a aplicar. La sesión requirió de aproximadamente 90 minutos por cada grupo escolar que colaboró en el estudio.

\section{Resultados}

La edad promedio de los alumnos fue de 19.9 años ( $D E=2.50$ ), 92\% entre 17 y 22 años; $28 \%$ de sexo masculino y $72 \%$ femenino; $93 \%$ solteros; $84 \%$ sin un empleo remunerado; $71 \%$ no contaban con beca, y $68 \%$ no había presentado exámenes extraordinarios en el semestre anterior.

La figura 1 muestra los porcentajes promedio de respuestas correctas, obtenidos por los alumnos de cada semestre académico, en el instrumento de comprensión lectora - considerando el puntaje total y el correspondiente a cada nivel de comprensión evaluado-. Las diferencias en el porcentaje de respuestas correctas, entre los alumnos de los distintos semestres, no rebasaron el 7\%, ni en el total del instrumento ni en cada nivel de comprensión. 


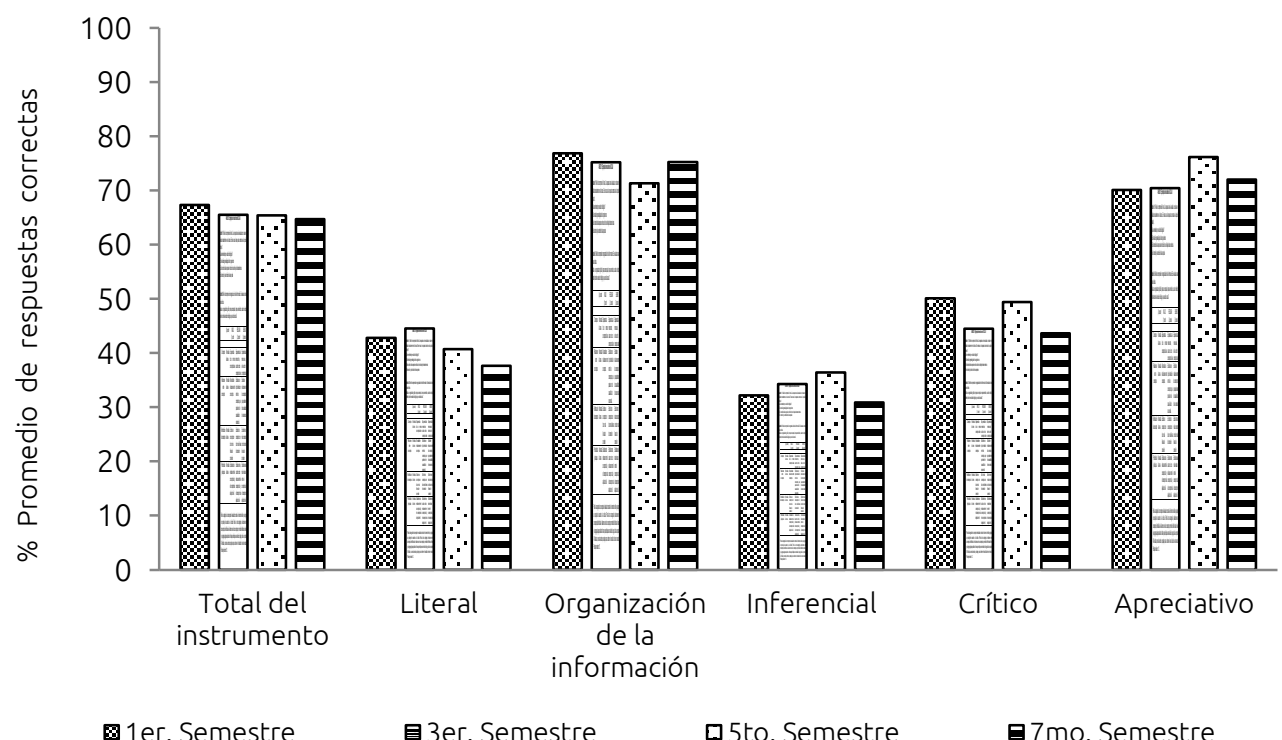

Figura 1. Porcentaje promedio de respuestas correctas obtenidas por los alumnos de cada semestre (total del ICLAU y en cada nivel de comprensión)

La aplicación de la prueba ANOVA indicó algunas diferencias estadísticamente significativas en distintos niveles de comprensión (ver tabla I), aunque no indicaron un patrón consistente a favor de los alumnos de un grado académico en particular.

Tabla I. Resultados del ANOVA para los niveles de comprensión por semestre académico

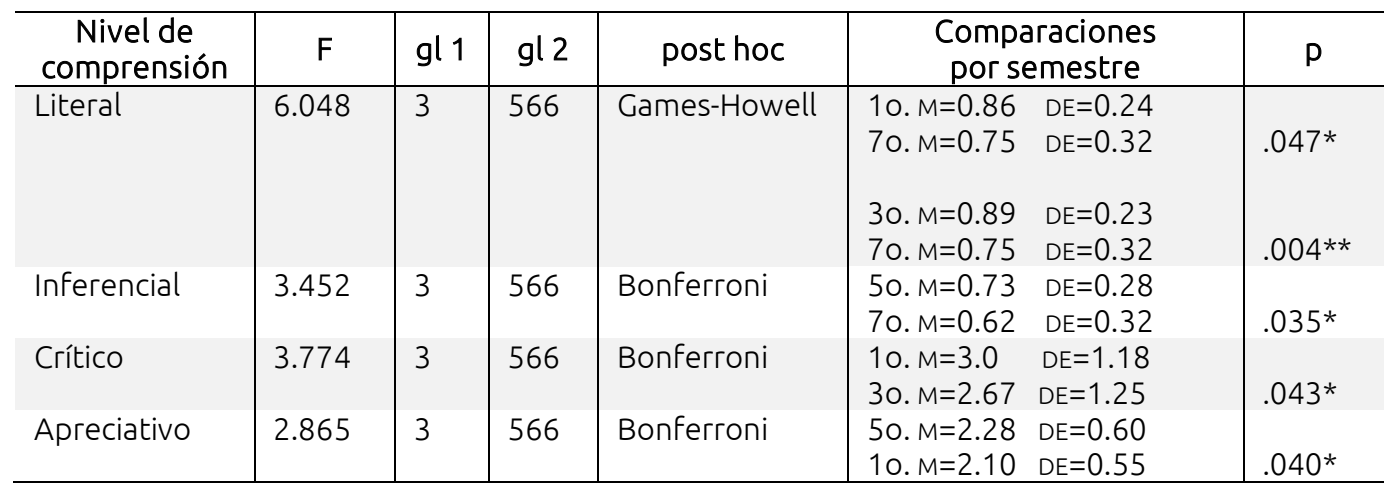

Nota: ${ }^{*} p<.05 ;{ }^{* *} p<.01$

Respecto a las estrategias lectoras y la motivación hacia la lectura, los promedios obtenidos en el total del inventario fueron similares para los alumnos de los distintos semestres, lo cual se vio reflejado en los valores de media (M) y desviación estándar ( $D E)$ : primer semestre ( $M=69$ y $D E=12.5)$, tercero ( $M=70$ y $D E=13.9)$, quinto $(M=70$ y $D E=14.3)$ y séptimo ( $M=68$ y $D E=15.0)$. Considerando la muestra total, las estrategias de Análisis de la lectura obtuvieron un 64\%, las de Identificación de información o ideas el 70\%, la Consulta de fuentes adicionales fueron las menos utilizadas (60\%) y la Motivación intrínseca hacia la lectura en general y hacia la lectura de textos académicos obtuvieron porcentajes similares (64\% y 62\%). La tabla II muestra que no hubo diferencias estadísticamente significativas en estos aspectos entre los estudiantes inscritos en cada semestre. 
Tabla II. Resultados del ANOVA para la comparación del IEMML por semestre académico

\begin{tabular}{lcrcc}
\hline Factores & $\mathrm{F}$ & $\mathrm{gl}$ 1 & $\mathrm{gl} 2$ & $\mathrm{P}$ \\
\hline Estrategias de análisis de lectura & 0.496 & 3 & 566 & .685 \\
Estrategias de identificación de la información o ideas & 0.122 & 3 & 566 & .947 \\
Estrategias de consulta de fuentes adicionales & 0.219 & 3 & 566 & .883 \\
Motivación intrínseca por la lectura en general & 0.739 & 3 & 566 & .529 \\
Motivación intrínseca por la lectura de textos académicos & 1.491 & 3 & 566 & .216 \\
\hline
\end{tabular}

En la figura 2 se observa que el mayor porcentaje de alumnos reportó dedicar entre 6 y 10 horas semanales a la lectura de textos académicos, mientras que el menor porcentaje indicó entre 21 y 25 horas. El ANOVA no arrojó diferencias entre los alumnos de distintos semestres $[F(3,566)=2.229, p=.084]$.

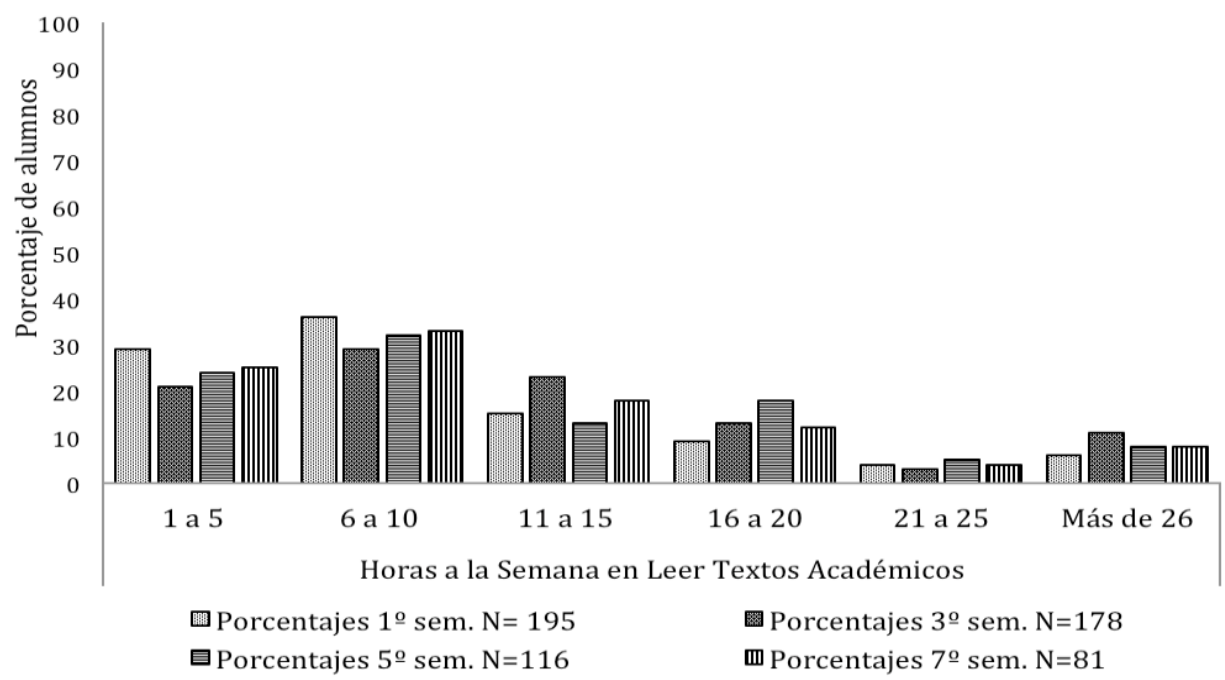

Figura 2. Porcentaje de alumnos, por semestre, que reportó cada rango de tiempo invertido semanalmente en la lectura de textos académicos

La tabla III muestra las relaciones entre la comprensión lectora del texto y diversas variables sociodemográficas y académicas. Fue mayor la comprensión en los alumnos que reportaron no tener un empleo remunerado que en quienes reportaron tenerlo ( $(567)=-2.20, p=.028)$; también fue mayor en los alumnos becados $[t(558)=2.16, p=.032]$. El ANOVA de un factor indicó diferencias en relación con el promedio académico $[\mathrm{F}=(4,565)=3.71, \mathrm{p}=.005]$; la prueba post hoc Bonferroni indicó que tal diferencia fue a favor de quienes tenían un promedio entre 9 y 10, respecto de los de 7 y 7.9 de promedio.

Tabla III. Resultados del ANOVA para el ICLAU y promedio académico

\begin{tabular}{|c|c|c|c|c|c|c|c|}
\hline$F$ & $\mathrm{gl1}$ & $\mathrm{gl2}$ & Post hoc & $\begin{array}{l}\text { Co } \\
\text { pro }\end{array}$ & $\begin{array}{l}\text { paracione } \\
\text { edio acad }\end{array}$ & $\begin{array}{l}\text { por } \\
\text { mico }\end{array}$ & $P$ \\
\hline 4.828 & 3 & 551 & Bonferroni & $\begin{array}{l}9.0-10 \\
7.0-7.9\end{array}$ & $\begin{array}{l}M=16.91 \\
M=15.90\end{array}$ & $\begin{array}{l}\mathrm{DE}=2.96 \\
\mathrm{DE}=2.91\end{array}$ & $.023^{*}$ \\
\hline
\end{tabular}

Nota: * $\mathrm{p}<.05$

En relación con las estrategias lectoras de los alumnos y su motivación hacia la lectura, se encontraron diferencias estadísticamente significativas a favor del sexo masculino $(t(556)=2.07, p=.039)$. Además, como se observa en la tabla IV, los alumnos con un promedio académico entre 9 y 10 tuvieron puntajes mayores que aquellos con promedios entre 7 y 7.9 , y entre 8 y $8.9[F(4,553)=3.02, p=.018]$. 
Tabla IV. Resultados del ANOVA para el IEMML y promedio académico

\begin{tabular}{|c|c|c|c|c|c|c|}
\hline$F$ & g 1 & $\mathrm{gl2}$ & Post hoc & $\begin{array}{l}\text { Com } \\
\text { Prom }\end{array}$ & $\begin{array}{l}\text { paraciones por } \\
\text { edio académico }\end{array}$ & $P$ \\
\hline 5.064 & 3 & 551 & Bonferroni & $\begin{array}{l}9.0-10 \\
7.0-7.9 \\
8.0-8.99\end{array}$ & $\begin{array}{l}M=73.79 \quad D E=14.12 \\
M=68.43 \quad D E=12.48 \\
M=68.42 \quad D E=13.83\end{array}$ & $\begin{array}{l}.010^{* *} \\
.002 * *\end{array}$ \\
\hline
\end{tabular}

Respecto al tiempo invertido en la lectura de textos académicos por parte de los alumnos, se observaron diferencias con relación en tres variables académicas. El ANOVA de un factor $[F(3,550)=5.409, p=.001]$ indicó que los alumnos con promedio entre 9 y 10 reportaron dedicar más tiempo que los alumnos con promedio entre 7 y 7.9. Las pruebas $t$ para muestras independientes arrojaron diferencias a favor de quienes contaban con beca [t(368)=-2.79, $\mathrm{p}=.006]$, y de quienes no habían presentado exámenes extraordinarios [t(558)=2.95, $\mathrm{p}=.003]$.

El ANOVA de un factor que analizó la relación entre el rango del tiempo invertido en la lectura de textos académicos y los puntajes totales del instrumento de comprensión lectora (ICLAU) no indicó diferencias significativas $[F(5,563)=0.375, p=.866]$. Sin embargo, como se observa en la tabla $V$, cuando se analizaron tales relaciones considerando cada uno de los niveles o tipos de comprensión lectora, se encontraron diferencias significativas en el nivel de comprensión literal, a favor de quienes reportaron leer textos académicos entre 16 y 20 horas.

Tabla V. ANOVA del ICLAU y el rango del tiempo invertido en la lectura

\begin{tabular}{|c|c|c|c|c|c|c|}
\hline $\mathrm{F}$ & $\mathrm{gl}$ & gl 2 & Post hoc & $\begin{array}{r}\text { Compar } \\
\text { de h }\end{array}$ & $\begin{array}{l}\text { raciones por rango } \\
\text { oras de lectura }\end{array}$ & $P$ \\
\hline 2.603 & 5 & 563 & Games-Howell & $\begin{array}{l}16 \text { a } 20 \\
1 \text { a } 5 \\
6 \text { a } 10\end{array}$ & $\begin{array}{ll}M=.93 & D E=.18 \\
M=.83 & D E=.28 \\
M=.81 & D E=.28\end{array}$ & $\begin{array}{l}.026^{*} \\
.000 * * *\end{array}$ \\
\hline
\end{tabular}

El ANOVA utilizado para relacionar el rango del tiempo invertido en la lectura de textos académicos con los puntajes del IEMML arrojó diferencias significativas $[F(5,563)=6.27, p=.000]$. En la tabla VI se observa que las diferencias fueron a favor de quienes reportaron dedicar mayor cantidad de horas a esta actividad.

Tabla VI. ANOVA para el IEMML y el rango de tiempo invertido en la lectura

\begin{tabular}{|c|c|c|c|c|c|c|c|}
\hline \multirow[b]{2}{*}{ IEMML } & \multirow{2}{*}{$\frac{F}{6.271}$} & \multirow{2}{*}{$\begin{array}{l}\text { gl1 } \\
5\end{array}$} & \multirow{2}{*}{$\frac{\mathrm{gl} 2}{563}$} & post hoc & \multicolumn{3}{|c|}{$\begin{array}{c}\text { Comparaciones por rango } \\
\text { de horas de lectura }\end{array}$} \\
\hline & & & & Bonferroni & $\begin{array}{l}1 \text { a } 5 \\
6 \text { a } 10 \\
11 \text { a } 15 \\
16 \text { a } 20 \\
21 \text { a } 25 \\
\text { Más de } 26\end{array}$ & $\begin{array}{l}M=66.13 \\
M=68.66 \\
M=69.37 \\
M=72.90 \\
M=76.24 \\
M=75.98\end{array}$ & $\begin{array}{l}D E=14.14 \\
D E=13.57 \\
D E=12.41 \\
D E=12.31 \\
D E=13.72 \\
D E=13.98\end{array}$ \\
\hline
\end{tabular}

Por último, para identificar el tipo de relación entre la comprensión lectora y las estrategias y motivación hacia la lectura, se utilizó el coeficiente de correlación producto-momento de Pearson, resultando una correlación positiva, aunque baja $(r=.108, N=570, p=.010)$ entre los puntajes de ambos instrumentos aplicados. 


\section{Discusión}

Los resultados obtenidos a través de la aplicación del instrumento de comprensión lectora permitieron observar que los alumnos de la muestra estudiada tuvieron un desempeño menor al $70 \%$. Aunque cabe recordar que el texto expositivo-argumentativo utilizado corresponde a un tema de Biología -y no de Psicología, que es la carrera que cursan-, esos niveles pueden considerarse bajos. Los menores desempeños se observaron, principalmente, en los niveles de comprensión literal, inferencial y crítica. Este hallazgo concuerda con lo reportado en investigaciones latinoamericanas (Cisneros et al., 2012; Echevarría, 2006 y Rizo, 2004), y confirma que los profesores universitarios enfrentan el problema de que sus alumnos presentan habilidades insuficientes para la comprensión, en especial para el análisis y síntesis de los textos que leen (Ugarriza, 2006). Si se parte de que la comprensión lectora debe darse en los alumnos universitarios como una competencia genérica, en todos los niveles evaluados, este parece ser un asunto que deben atender los planes curriculares universitarios.

En lo relativo a la aplicación del Inventario de Estrategias Metacognoscitivas y Motivación, el nivel promedio obtenido (64\%) también se considera bajo. Las estrategias más utilizadas fueron las de identificación de información o ideas, y las menos utilizadas, las de consulta de fuentes adicionales. Si se toma en cuenta que estos datos sí guardan una relación directa con los textos expositivo-argumentativos que estudian durante su carrera, puede decirse que estos aspectos también son asuntos a atender por parte de las universidades.

En otro rubro, los datos indicaron que el mayor porcentaje de la muestra reportó dedicar entre 6 y 10 horas a la lectura de textos académicos, lo que concuerda con lo reportado en estudios previos (De Garay, 2004). Tiempo que no puede considerarse suficiente para cumplir con las materias que cursan por semestre. Dicha variable resultó ser sumamente importante en esta investigación porque los análisis indican que los alumnos que reportaron dedicar más tiempo obtuvieron mayores puntajes en la comprensión lectora de tipo literal, pero principalmente porque se asoció positivamente con el uso de estrategias metacognoscitivas y con la motivación hacia la lectura. Tal resultado es compatible con otras evidencias sobre el tema (Susar, 2010), y tiene sentido si se considera que entre más oportunidades tiene el estudiante de enfrentarse con los textos puede hacer mayores esfuerzos por entender lo que lee, y mayores serán las probabilidades de que use las estrategias que ha adquirido con anterioridad. Aunque hay que considerar también que ello no necesariamente garantiza un incremento en la comprensión lectora en todos los niveles y que, aun cuando el estudiante utilice una o varias estrategias, no necesariamente las utiliza de manera óptima. Estas consideraciones hacen necesaria la realización de estudios empíricos sobre la forma en que los alumnos universitarios utilizan cada una de las estrategias para leer sus textos académicos.

Respecto a las variables sociodemográficas y académicas que se asociaron con la comprensión lectora, y con el uso de estrategias y motivación, figuran: que los alumnos no trabajen, que tengan un promedio alto, y contar con beca. Tales relaciones son esperables y coherentes porque, al no tener obligaciones laborales, pueden dedicar más tiempo al estudio y al desarrollo de estrategias, lo que repercute en su promedio académico y en conseguir una beca (Ugarriza, 2006).

Los hallazgos también confirman una correlación positiva entre la comprensión lectora de un texto expositivo-argumentativo, de corte académico, y el uso de estrategias y motivación hacia la lectura (Mézquita, Benois e Yver, 2010).

Los datos más importantes arrojados por la presente investigación se relacionan con el hecho de no haber encontrado diferencias estadísticamente significativas entre los estudiantes que cursan los diferentes semestres de la carrera, en ninguno de los aspectos evaluados. Esto puede considerarse contrario a lo deseable porque, a medida que avanzan en su carrera profesional tendrían que mostrar: un mayor grado de competencia en comprensión lectora, en todos los niveles implicados; un uso de estrategias lectoras más variado y encaminado a obtener más información de sus textos académicos; una mayor motivación intrínseca hacia la lectura de textos generales y académicos, y dedicar mayor número de horas semanales al estudio. Tal hallazgo puede guardar una estrecha relación con las competencias promovidas por las 
propias universidades. En un estudio realizado en México (Vera, Estévez y Ayón, 2010), los alumnos reconocen que carecen de habilidades de abstracción, análisis, síntesis y crítica, atribuyéndolo al hecho de que los programas académicos de sus universidades no están dirigidos a fomentar tales habilidades, aunque también reconocen lo poco que ellos se esfuerzan por lograrlas.

De lo anterior se puede concluir que los contenidos curriculares y el desarrollo académico que siguen los estudiantes conforme avanzan en sus grados escolares no parecen tener impacto sobre su comprensión, su uso de estrategias metacognoscitivas ni sobre su motivación hacia la lectura de textos académicos.

Los datos parecen indicar la necesidad de que se implementen cursos, programas y talleres propedéuticos que enseñen a los estudiantes, en los distintos niveles educativos, prácticas efectivas para la comprensión lectora, el desarrollo de estrategias y otras prácticas relacionadas con el desempeño académico y la motivación. Serrano (2008) menciona que la formación de estudiantes universitarios debe contemplar el entrenamiento en la lectura crítica y multidisciplinaria, con el fin de incrementar su capacidad de evaluar y construir juicios, para tomar decisiones y posturas con respecto a un tema en particular. Mientras que Echevarría (2006) y Edmonds et al. (2009) demuestran que los estudiantes con problemas para la comprensión lectora pueden mejorar sus habilidades cuando se les enseñan explícitamente prácticas enfocadas a ello. Lo anterior parece obvio, pero lo estudiantes no suelen ser evaluados en estas habilidades y estrategias.

Considerando los resultados aquí reportados, las aportaciones en cuanto al estudio de la competencia genérica denominada comprensión lectora, para la población estudiada, son importantes en el sentido de que ejemplifican una parte de las habilidades con las que los estudiantes cuentan para enfrentar los requerimientos académicos del plan de estudios, así como los laborales más inmediatos. Por lo tanto, en los diseños de nuevos planes de estudio de la carrera de Psicología, y probablemente de muchas otras, debiera ser considerada la competencia genérica de la comprensión lectora como potenciadora de aprendizaje, con el objetivo de formar a las nuevas generaciones con bases cada vez más sólidas que les auxilien a mejorar su rendimiento académico y laboral.

\section{Referencias}

Anmarkrud, O. y Braten, I. (2009). Motivation for reading comprehension. Learning and Individual Differences, 19(2), 252-256. doi:10.1016/j.lindif.2008.09.002

Carlino, P. (2007). El problema de leer textos complejos al comienzo de la universidad: situaciones didácticas para hacerle frente. Impulso, 1, 9-16.

Cisneros, M., Olave, G. y Rojas, I. (2012). Cómo mejorar la capacidad inferencial en estudiantes universitarios. Educación y Educadores, 15(1), 45-61.

De Garay, A. (2004). Los actores desconocidos. Una aproximación al conocimiento de los estudiantes. México: ANUIES.

Echevarría, M. A. (2006). ¿Enseñar a leer en la universidad? Una intervención para mejorar la comprensión de textos complejos al comienzo de la educación superior. Revista de Psicodidáctica, 11(2), 169-188.

Edmonds, S., Vaughn, S., Wexler, J., Reutebuch, C., Cable, A., Klingler, K. y Wick, J. (2009). A synthesis of reading interventions and effects on reading comprehension outcomes for older struggling readers. Review of Educational Research, 79(1), 262-300.

Guerra, J. y Guevara, Y. (2013). Validación de un instrumento para medir comprensión lectora en alumnos universitarios mexicanos. Enseñanza e Investigación en Psicología, 18(2), 277-291. 
Guerra, J., Guevara, Y. y Robles, S. (2014). Validación del Inventario de Estrategias Metacognoscitivas y Motivación por la Lectura (IEMML) en estudiantes de psicología. USB Revista Psicogente, 17(31), 17-32.

Instituto Nacional para la Evaluación de la Educación (2009). Información sobre México en Pisa 2009. México: Autor.

Méndez, I., Namihira, D., Moreno L. y Sosa, C. (2006). El protocolo de investigación. Lineamientos para su elaboración y análisis. México: Trillas.

Mézquita, N., Benois, N. e Yver, T. (2010). Comprensión de textos y estrategias de aprendizaje. Revista Científica Electrónica de Psicología, 10, 185-200.

Mujica, A., Guido, P. y Mercado, S. (2011). Actitudes y comportamiento lector: una aplicación de la teoría de la conducta planeada en estudiantes de nivel medio superior. Liberabit, 17(1), 77-84.

Oliver, K. (2009). An investigation of concept mapping to improve the reading comprehension of science texts. Journal of Science Education \& Technology, 18(5), 402-414.

Organización para la Cooperación y el Desarrollo Económicos. (2002). Resultados de PISA 2000. Programa Internacional de Evaluación de Estudiantes. México: Aula XXI, Santillana.

Peña, J. (2000). Las estrategias de lectura: su utilización en el aula. Educere, 4(11), 159-163.

Pérez, J. (2005). Evaluación de la comprensión lectora: Dificultades y limitaciones. Revista de Educación, número extraordinario, 121-138.

Rizo, M. (2004). Programa de instrucción para desarrollar estrategias para la comprensión y el aprendizaje de textos escritos. Revista Latinoamericana de Estudios Educativos, 34(2), 113-137.

Sánchez, E. y Acle, G. (2001). Relación entre comprensión lectora y niveles de pensamiento en estudiantes universitarios. Enseñanza e Investigación en Psicología, 6(2), 225-241.

Serrano, S. (2008). El desarrollo de la comprensión crítica en los estudiantes universitarios. Educere, $12(42), 505-514$.

Silvers, V., Kreiner, S. y Natz-González, A. (2009). Harmful effects of preexisting inappropriate highlighting on reading comprehension and metacognitive accuracy. The Journal of General Psychology, 136(3), 287300.

Susar, F. (2010). Relationship between reading comprehension strategy use and daily free reading time. Procedia Social and Behavioral Sciences, 2, 4752-4756.

Taboada, A., Tonks, M., Wigfield, A. y Guthrie, T. (2009). Effects of motivational and cognitive variables on reading comprehension. Read Write: An Interdisciplinary Journal, 22, 85-106.

Ugarriza, N. (2006). La comprensión lectora inferencial de textos especializados y el rendimiento académico de los estudiantes universitarios del primer ciclo. Persona, 9, 31-76.

Vera, A., Estévez, E. y Ayón, L. (2010). Percepción de estudiantes universitarios sobre importancia y realización de competencias genéricas. Revista de Educación y Desarrollo, 15, 47-54.

Wang, D. (2009). Factors affecting the comprehension of global and local main idea. Journal of College Reading and Learning, 39(2), 34-51. 
Washer, P. (2007). Revisiting key skills: A practical framework for higher education. Quality in Higher Education, 13(1), 57-67.

Zhang, J. y Wu, A. (2009). Chinese senior high school EFL students' metacognitive awareness and readingstrategy use. Reading in a Foreign Language, 21(1), 37-59. 


\section{ANEXO 1. Ejemplos de reactivos del ICLAU}

Reactivo 1: Nivel de comprensión literal. La respuesta será evaluada con base en lo señalado textualmente en la lectura. En este caso la respuesta correcta es la opción a. Puntaje: 1.

¿Qué se entiende por evolución biológica?

a) Es la relación genealógica de los organismos

b) Es el cambio de las especies en función de un linaje de descendencia

c) Es el cambio y la extinción de las especies

Reactivo 3: Nivel de comprensión: reorganización de la información. Es evaluado a través de una rúbrica.

"Realiza un organizador gráfico (mapa conceptual, mapa semántico, cuadro sinóptico, etc.) sobre la evolución biológica y su estudio actual".

\begin{tabular}{|c|c|c|c|c|}
\hline & (0 puntos) & $\begin{array}{c}\text { MALO } \\
\text { (1 punto) }\end{array}$ & $\begin{array}{l}\text { REGULAR } \\
\text { (2 puntos) }\end{array}$ & $\begin{array}{l}\text { BUENO } \\
\text { (3 puntos) }\end{array}$ \\
\hline Conceptos & $\begin{array}{l}\text { No realiza } \\
\text { la tarea }\end{array}$ & $\begin{array}{l}\text { Esquematiza dos o menos } \\
\text { conceptos clave. }\end{array}$ & $\begin{array}{l}\text { Esquematiza al menos } \\
\text { tres, cuatro o cinco } \\
\text { conceptos clave. }\end{array}$ & $\begin{array}{l}\text { Esquematiza al menos } \\
\text { seis, siete u ocho } \\
\text { conceptos clave. }\end{array}$ \\
\hline $\begin{array}{l}\text { Relaciones } \\
\text { entre } \\
\text { conceptos }\end{array}$ & $\begin{array}{l}\text { No realiza } \\
\text { la tarea }\end{array}$ & $\begin{array}{l}\text { No establece relaciones } \\
\text { entre conceptos. }\end{array}$ & $\begin{array}{l}\text { Establece un tipo de } \\
\text { relación entre los } \\
\text { conceptos, que puede ser } \\
\text { de causalidad o secuencial. }\end{array}$ & $\begin{array}{l}\text { Establece relaciones } \\
\text { entre los conceptos que } \\
\text { pueden ser de causalidad } \\
\text { o de secuencia. }\end{array}$ \\
\hline $\begin{array}{l}\text { Ramificación } \\
\text { de conceptos }\end{array}$ & $\begin{array}{l}\text { No realiza } \\
\text { la tarea }\end{array}$ & $\begin{array}{l}\text { Escribe un concepto con } \\
\text { dos o más líneas de } \\
\text { conexión }\end{array}$ & $\begin{array}{l}\text { Escribe dos conceptos } \\
\text { con dos o más líneas de } \\
\text { conexión }\end{array}$ & $\begin{array}{l}\text { Escribe tres o más } \\
\text { conceptos con dos o } \\
\text { más líneas de conexión }\end{array}$ \\
\hline $\begin{array}{l}\text { Profundidad } \\
\text { jerárquica }\end{array}$ & $\begin{array}{l}\text { No realiza } \\
\text { la tarea }\end{array}$ & $\begin{array}{l}\text { Establece dos enlaces } \\
\text { entre el concepto raíz y } \\
\text { el concepto más alejado } \\
\text { de él. }\end{array}$ & $\begin{array}{l}\text { Establece tres, cuatro } \\
\text { o cinco enlaces entre el } \\
\text { concepto raíz y el } \\
\text { concepto más alejado } \\
\text { de él. }\end{array}$ & $\begin{array}{l}\text { Establece seis o más } \\
\text { enlaces entre el concepto } \\
\text { raíz y el concepto más } \\
\text { alejado de él. }\end{array}$ \\
\hline
\end{tabular}

Para la categoría de conceptos el evaluador contará el número de ellos y asignará un puntaje de acuerdo a la rúbrica. La otra categoría (relaciones entre conceptos) identificará si el alumno une los conceptos por medio de flechas o líneas, y si agrega alguna palabra o frase que indique una relación; luego, dará un puntaje. Al finalizar, sumará ambos puntajes para obtener el resultado de este reactivo. Puntaje máximo: 12. 
ANEXO 2. Ejemplos de reactivos del IEMML

\begin{tabular}{|c|c|c|c|c|c|}
\hline $\begin{array}{l}\text { 7. Cuando leo, busco el significado de las } \\
\text { palabras } \\
\text { que no entiendo. }\end{array}$ & NUNCA & RARA VEZ & A VECES & FRECUENTEMENTE & SIEMPRE \\
\hline $\begin{array}{l}\text { 12. Cuando leo puedo identificar las ideas del } \\
\text { autor. }\end{array}$ & NUNCA & RARA VEZ & A VECES & FRECUENTEMENTE & SIEMPRE \\
\hline 14. Leo por iniciativa propia. & NUNCA & RARA VEZ & A VECES & FRECUENTEMENTE & SIEMPRE \\
\hline $\begin{array}{l}\text { 22. Al terminar la lectura puedo dar una } \\
\text { explicación verbal de lo leído. }\end{array}$ & NUNCA & RARA VEZ & A VECES & FRECUENTEMENTE & SIEMPRE \\
\hline
\end{tabular}

